\title{
Which Aesthetic Has the Greatest Effect on Human Understanding?
}

\author{
Helen Purchase \\ Department of Computer Science and Electrical Engineering, \\ The University of Queensland, Australia
}

\begin{abstract}
In the creation of graph drawing algorithms and systems, designers claim that by producing layouts that optimise certain aesthetic qualities, the graphs are easier to understand. Such aesthetics include maximise symmetry, minimise edge crosses and minimise bends.
\end{abstract}

A previous study aimed to validate these claims with respect to three aesthetics, using paper-based experiments [11]. The study reported here is superior in many ways: five aesthetics are considered, attempts are made to place a priority order on the relative importance of the aesthetics, the experiments are run on-line, and the ease of understanding the drawings is measured in time, as well as in the number of errors. In addition, greater consideration is given to the possible effect of confounding factors in the graph drawings.

The results indicate that reducing the number of edge crosses is by far the most important aesthetic, while minimising the number of bends and maximising symmetry have a lesser effect. The effects of maximising the minimum angle between edges leaving a node and of fixing edges and nodes to an orthogonal grid are not statistically significant.

This work is important since it helps to demonstrate to algorithm and system designers the aesthetic qualities most important for aiding human understanding, the most appropriate compromises to make when there is a conflict in aesthetics, and consequently, how to build more effective systems.

\section{Introduction}

Automatic graph drawing algorithms produce a diagram which represents an underlying graph structure. The aim of the layout process is to depict relational information in a form that makes it easier to read, understand and use. Designers of such algorithms ensure that certain aesthetics are optimised, and claim that by doing do, the resultant graph drawing helps the human reader to understand and remember the information embodied in the graph. Examples of these aesthetics include: symmetry (where possible, a symmetrical view of the graph should be displayed [5, 10]), minimise edge crosses (the number of edge crosses in the display should be minimised [6]), and minimise bends (the total number of bends in polyline edges should be minimised $[13,15])$. 
It is important that human experiments be performed on these aesthetics, so that, rather than judging an algorithm by its computational efficiency in conforming to these aesthetics, the aesthetics themselves can be judged with respect to how much they assist human comprehension. Many application domains may make use of automatic graph layout algorithms in order to display relational data in a holistic form: e.g. entity relationship diagrams [1], object oriented design diagrams [4], social networks [3]. If the designers of automatic graph layout algorithms are to claim that their algorithms will illuminate the information embodied therein, it is important that they know that the aesthetic basis for their work is sound.

Many algorithms consider more than one aesthetic in their attempt to create an illuminating graph drawing. For this reason, although the individual aesthetics themselves are important, often it is the combination or prioritisation of the aesthetics that is most useful. Algorithm designers may need to compromise between more than one aesthetic. For example, in the creation of a particular drawing, minimising the number of crosses may also result in a decrease in symmetry. The knowledge that minimising the number of crosses is of more benefit to understandability than maximising symmetry [11], means that an appropriate compromise can be made.

The previous study performed preliminary paper-based experiments on the human understanding of graph drawings to determine whether three aesthetic criteria (crosses, bends and symmetry) did indeed assist with the understanding of the underlying graph structure. While the hypotheses were confirmed in the case of crosses and bends, there was not enough evidence to either support or reject the symmetry hypothesis.

In this experiment, five aesthetics were considered; there are therefore five primary hypotheses:

- Bends (b):

Increasing the number of edge bends in a graph drawing decreases the understandability of the graph.

- Crosses (c):

Increasing the number of edge crosses in a graph drawing decreases the understandability of the graph.

- Angles (m):

Maximising the minimum angle between edges leaving the nodes in a graph drawing increases the understandability of the graph.

- Orthogonality (o):

Fixing nodes and edges to an orthogonal grid increases the understandability of the graph.

- Symmetry (s):

Increasing the symmetry displayed in a graph increases the understandability of the graph. 
Briefly, the experiment entailed subjects answering questions about a number of different drawings of the same graph. Each drawing was drawn such that it varied the aesthetics under consideration in a fixed manner: for example, one drawing had a large number of crosses, while another had less. Measurements were taken of both the number of errors made and the time taken to answer the questions. Using statistical tests, the five primary hypotheses associated with the five different aesthetics under consideration were proved or disproved. In addition, both for the set of "easy" drawings as well as the set of "difficult" drawings, Tukey's WSD pairwise comparison procedure was then used to determine if there were significant understandability priorities between the aesthetics.

Experiments were run online to study these five aesthetics, and the results indicate that crosses is by far the most important aesthetic. Bends and symmetry have a lesser effect, and maximising the minimum angle and maximising orthogonality have no significant effect at all. This paper describes the nature of the on-line system used for the experiments and the experimental methodology (the graph drawings, experiment and the data), and presents and discusses the results.

\section{The Experiment}

\subsection{Definition}

There are two ways in which understandability may be measured. A purely relational method measures the efficiency and accuracy with which people can read a graph structure and answer questions about it. Such graph-theoretic questions need to be generic and application-independent, and may include questions of the form "What is the shortest path from node A to node B?" A more applicationspecific method would rather consider a graph interpretation task: in this case it is more appropriate that the effectiveness of the graph drawing is measured within the context in which the application-specific graph is usually used. Thus, instead of eliciting answers to specific questions asked about the graph itself, it is more suitable to look at whether the graph has assisted the user in accomplishing a particular application task. Suitable questions for this approach would include (in the area of software engineering) "What object classes would be affected by changing the external interface to class X?"

In this experiment, the relational reading of a graph drawing is considered, leaving the interpretive consideration of aesthetics for a later study. The questions that are used in this experiment to measure relational understandability are:

- How long is the shortest path between two given nodes?

- What is the minimum number of nodes that must be removed in order to disconnect two given nodes such that there is no path between them?

- What is the minimum number of edges that must be removed in order to disconnect two given nodes such that there is no path between them? 


\subsection{Scope}

A preliminary, more limited, study [11] reported comparable conclusions to those reported here. The study reported here improves on this previous study in a number of important ways, greatly increasing the validity and relevance of the results:

- Metric definitions: New metrics for all five aesthetics have been defined [12]. These are all scaled to lie between 0 and 1 , where 0 represents an amount of the aesthetic that it is assumed makes the drawing difficult to read (e.g. not much orthogonality), while 1 represents an amount of the aesthetic that it is assumed makes the drawing easy to read (e.g. not many crosses). A new metric for symmetry has been defined, which more closely represents perceptual symmetry than the one used previously. It takes into account both global and local symmetries, weighting them by their area, and also considers the effects of crosses and bends on perceptual symmetry.

- Presentation medium: The experiments are performed online using an experimental system especially designed and implemented for experiments like these. This means that the understandability of the graph drawings is tested using a more valid medium: automatic graph layout algorithms by definition make use of a computer, with the results displayed on a screen, rather than on paper. Experiments where subjects read graph drawings on a screen are therefore more valid than similar paper-based experiments.

- Dependent variables: The use of the online system enables two dependent variables to be recorded: the time taken for the subject to answer the question (the "reaction time"), as well as the correctness of the answer. This enables analysis to be performed on two measures of understanding.

- Confounding factors: In the drawings that vary a particular aesthetic, it is important that the values of the other four aesthetics are kept constant, to ensure that there is no confounding of variables. It is difficult, and in some cases impossible, to use the extremes of 0 or 1 as the constant value for the other four aesthetics: for example, a metric value of 0 for the bend aesthetic would imply a maximum possible number of bends; a metric value of 1 for minimum angle aesthetic would mean that all nodes in the drawing have the optimum angles between its edges (impossible for any cyclic graph). For this reason, a "neutral range" was defined for each aesthetic (based on perception), and for the drawings which varied a particular aesthetic, values of the other four aesthetics were kept within these specified ranges.

- Location of nodes: The questions that are asked about the drawings refer to nodes that are highlighted in black on the screen, to distinguish them from the other nodes. The relevant nodes are therefore obvious to the subjects, and the time measured for the subject to answer the question does not include additional time taken for locating the important nodes. The previous study referred to the nodes by labels [11]. 


\subsection{The Online System}

Experiments were run online. Each subject interacted with a unique experimental program. These programs were created by a system designed and implemented for the purposes of running experiments relating to graph drawings (called SAGE). The main features of SAGE are:

- Flexibility: so that SAGE can be used for further graph-drawing experimentation, each experiment is specified with an external contents file.

- Randomness: the ordering of graph drawings, their orientation, the ordering of the questions, and the selection of node-pairs for the questions are all able to be randomised.

- Graph and question flexibility: the graph drawings and questions used are defined in separate files, and are easily changed. ${ }^{1}$

- Completeness: all the interface features required for each graph drawing display are provided and specified in the contents file: text, pictures, input fields, pushbuttons.

- Robustness: SAGE can withstand the unexpected input of a novice user, and efficiently and correctly represents the experiment as defined in the contents file.

- Analysable data: the results for each subject are generated automatically as a list of the time between the display of each drawing and question and the entry of an answer, the answer itself, and its correctness.

\subsection{The Graphs}

The graph for this experiment was carefully designed so that node-pairs could be identified which gave a suitable range of values for the three questions. Thus, a set of node-pairs was defined that would give correct answers to the first question (the shortest path) of either 2,3,4 or 5; a set of node-pairs was defined that would give correct answers to the second question (the number of nodes to remove) of either 1 or 2; and a set of node-pairs was defined that would give correct answers to the third question (the number of edges to remove) of either 1,2 or 3 . The graph has 16 nodes and 28 edges.

New metric formulae (all lying within the range 0 to 1 ) were defined for this experiment, including a more extensive definition of symmetry [12]. Ten experimental graphs were created, two for each of the aesthetics (representing a strong or weak presence of the aesthetic). For convenience, the graph drawings are called after the aesthetic that they consider $(\mathbf{b}, \mathbf{c}, \mathbf{m}, \mathbf{o}, \mathbf{s})$, and + or - depending on the strength of the aesthetic: + indicates a high aesthetic value (i.e. assumed to be easy to read), and - indicates a low aesthetic value (i.e. assumed to be

\footnotetext{
${ }^{1}$ The graph drawings are in GRAPHED format [8], and the questions are in ASCII.
} 
difficult to read). Thus, the $\mathbf{s}+$ drawing has a symmetry metric value closer to 1 than the s- drawing.

Figures 1 and 2 show the ten graph drawings, and their associated metric values. Note that because of the nature of the aesthetics, the metrics cannot be sensibly compared over the aesthetic dimension. Thus, while c- has a cross-less value of $0.87, \mathbf{m}$ - has a value of $0.16 ; \mathbf{s}+$ has a symmetry value of $0.96, \mathbf{o}+$ has an orthogonality value of 0.46 . This variation is due to the metric definitions and distributions: it does not affect the results, as the important feature is the variation of the values within the aesthetic dimension. ${ }^{2}$

Due to the careful manipulation of aesthetics that was required, some of these drawings may look strangely awkward (e.g. b-, m-). As the aim was to consider the effect of the individual aesthetics (rather than drawings that may feasibly be produced by layout algorithms, or that have been purposefully drawn "neatly"), the artificial nature of some of the drawings was both intentional and necessary.

\subsection{Experimental Methodology}

The structure of the experiment was similar to the previous paper-based preliminary investigation [11]. The contents file used by SAGE defined experimental programs of the following form:

1. A brief description of graphs, and definitions of the terms node, edge, path, and path length were presented, followed by an explanation of the three questions that the subjects were required to answer about the experimental graphs. A simple example graph drawing, with the three questions and their correct answers, was shown. At this stage, the subjects were asked if they had any questions about graphs in general, or about the experiment. It was important to ensure that all the subjects knew what was expected of them.

2. The three questions were asked of six "practise" graph drawings, to familiarise the subjects with the nature of graph drawings and the questions, and to ensure that they were comfortable with the task, before tackling the experimental graphs. The subjects were not told that these graph drawings were not experimental.

3. A "filler" task which engaged the subjects" mind on a small problem unrelated to graphs was presented. This ensured that their performance on the subsequent experimental graphs was not affected by any follow-on effect from the practise graphs. A simple logic puzzle, designed to take approximately 1 minute, was used.

4. The ten experimental graph drawings were each displayed three times, once for each question. The order of presentation of the drawings and the questions was random, as was the orientation of the drawings.

${ }^{2}$ The metric definitions give more detail on the extremes of the metric values [12]. 


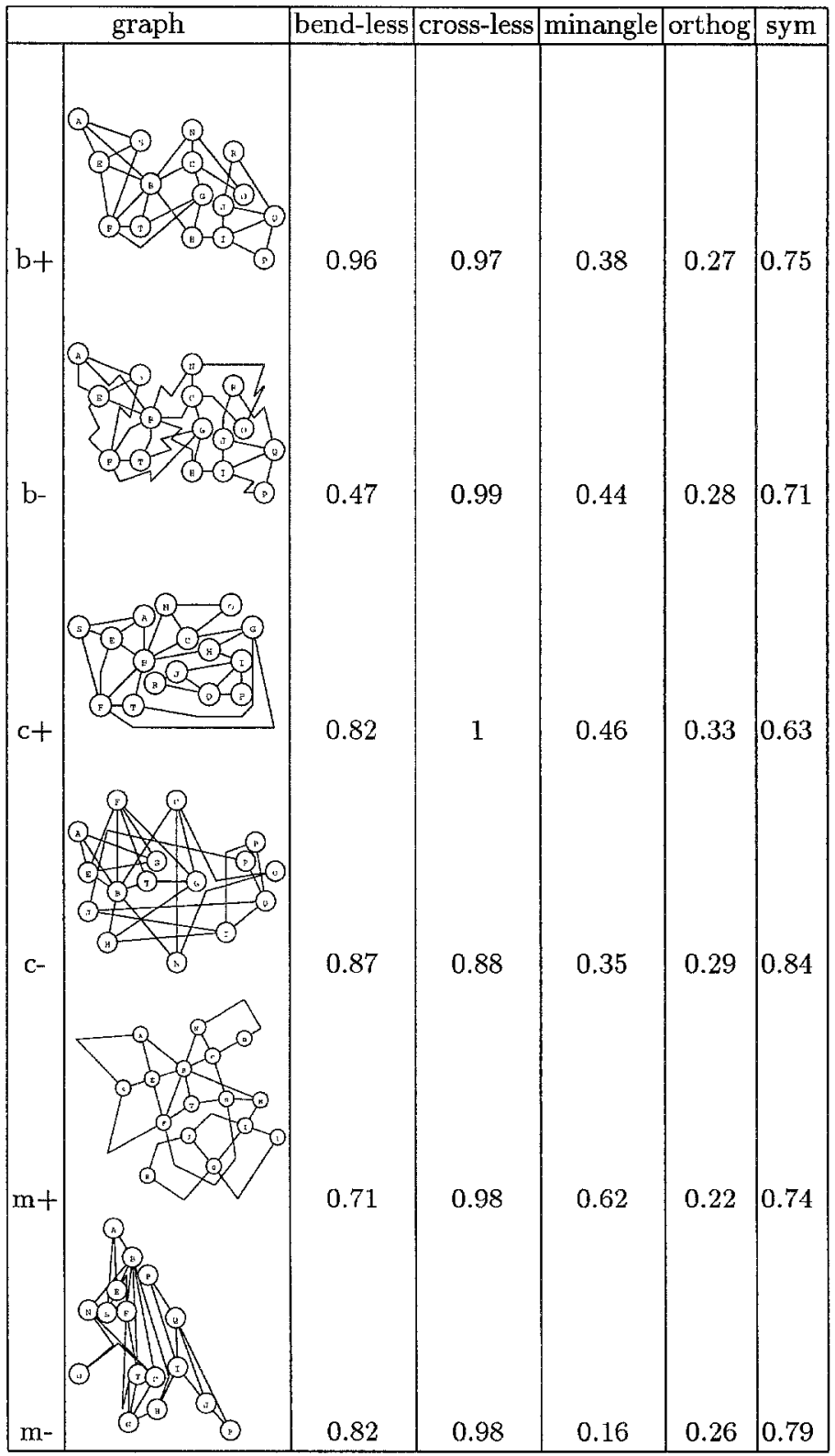

Fig. 1. Six of the ten experimental graph drawings, and their aesthetic values. 


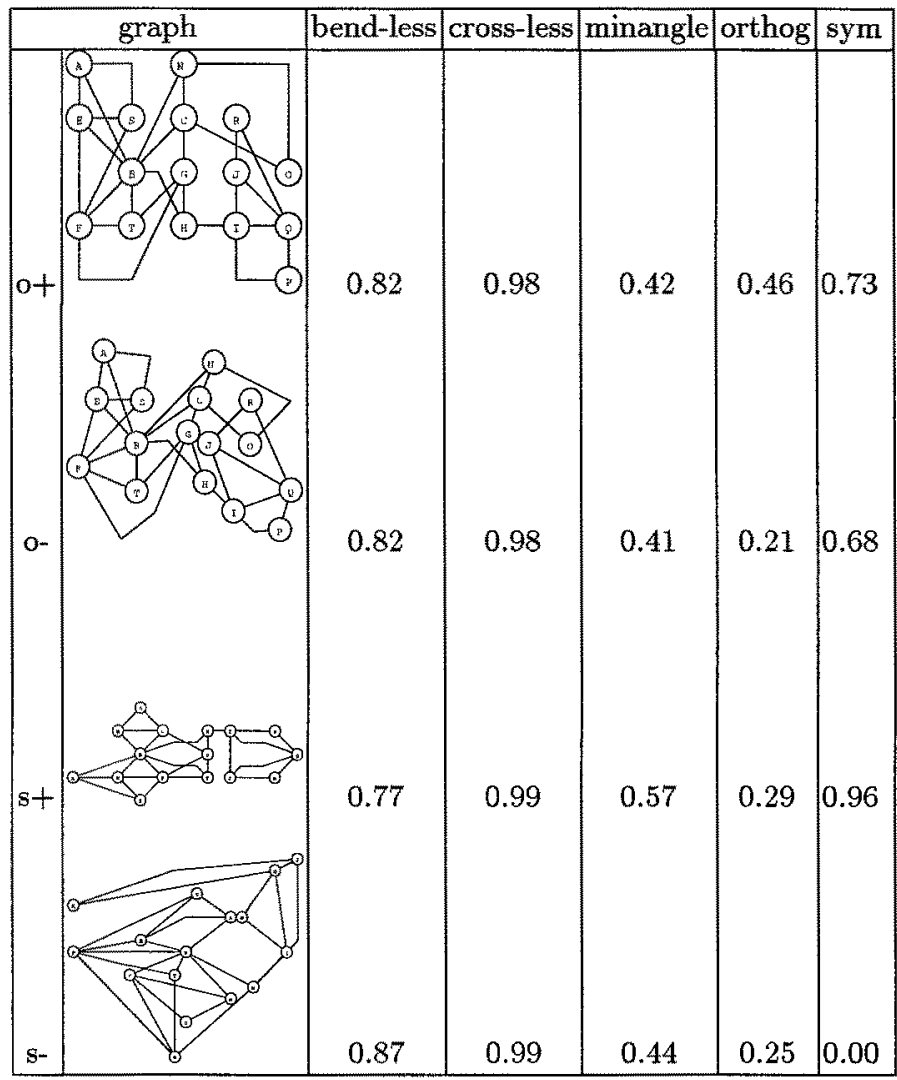

Fig. 2. Four of the ten experimental graph drawings, and their aesthetic values.

The questions themselves were randomised too: although the same three questions were asked of each drawing, the pair of nodes chosen for each question was randomly selected from a list of node-pairs (as defined in an external question file). This ensured that any variability in the data could not be explained away by the varying difficulty of the questions. The two relevant nodes for each question were highlighted in black on the screen, ensuring that reaction time did not include time taken to locate the nodes.

The subjects typed their answers to the questions: the time taken for their answer, and the correctness of the answer, was recorded.

The experiment was therefore controlled for the questions and the graphs, the independent variable was the value of the aesthetics in each drawing, and the two dependent variables were the time taken to answer the questions, and the number of errors made for each drawing. 
A within-subjects analysis method was used in order to reduce any variability that may have been attributable to the difference between the subjects (e.g. age, experience). Any learning effect was minimised by the large number of graphs used in the experiment, the inclusion of the practise graphs, and the randomisation of the ordering of the graph drawings.

55 second-year computer science students at The University of Queensland took part in the experiment, for a reward of $\$ 10$. For each subject and for each drawing, the total number of errors was recorded, as well as the total time taken to answer all three questions.

\section{Results}

The average number of errors and the average reaction time for the ten experimental graph drawings are shown in both tabular and chart form in Fig. 3.

\subsection{Testing the Five Individual Hypotheses}

To test the five primary hypotheses, one for each aesthetic, first the significance of the effects of the level of difficulty (the $+/-$ dimension) needed to be confirmed. After this confirmation that the $+/-$ dimension had indeed affected the error and reaction time data collected, each individual aesthetic was then tested for its contribution to this overall effect. This analysis was performed for both errors and reaction time.

Results. The $2 \times 5$ within-subject analysis of variance showed that: ${ }^{3}$

- The main effect of the level of difficulty (the $+/$ - dimension) was significant for both errors $\left(F_{1,54}=14.89, \alpha=.05\right)$ and reaction time $\left(F_{1,54}=40.67, \alpha=.05\right)$.

- The simple effect of the bends metric was significant for errors $\left(F_{1,54}=14.49, \alpha=.01\right)$ but only approaches significance for reaction time $\left(F_{1,54}=5.84, \alpha=.01\right)$.

- The simple effect of the crosses metric was significant for both errors $\left(F_{1,54}=24.25, \alpha=.01\right)$, and reaction time $\left(F_{1,54}=87.98, \alpha=.01\right)$.

- The simple effect of the minimum angle metric was not significant for both errors $\left(F_{1,54}=0.09, \mathrm{NS}\right)$ and reaction time $\left(F_{1,54}=3.05, \mathrm{NS}\right)$.

- The simple effect of the orthogonality metric was not significant for both errors $\left(F_{1,54}=0.00, \mathrm{NS}\right)$ and reaction time $\left(F_{1,54}=1.44, \mathrm{NS}\right)$.

- The simple effect of the symmetry metric was not significant for errors $\left(F_{1,54}=0.09, \mathrm{NS}\right)$, but was significant for reaction time $\left(F_{1,54}=7.57, \alpha=.01\right)$.

${ }^{3}$ The statistical analysis used here is a standard ANOVA analysis [9], based on the critical values of the $\mathrm{F}$ distribution: $\alpha$ is the level of significance, and results that are not significant are indicated by NS. 


\begin{tabular}{|l|l|l|l|l|l|l|l|l|l|l|}
\hline & $\mathrm{b}+$ & $\mathrm{b}-$ & $\mathrm{c}+$ & $\mathrm{c}-$ & $\mathrm{m}+$ & $\mathrm{m}-$ & $\mathrm{o}+$ & $\mathrm{o}-$ & $\mathrm{s}+$ & $\mathrm{s}-$ \\
\hline errors & 0.24 & 0.53 & 0.29 & 0.80 & 0.36 & 0.38 & 0.36 & 0.36 & 0.29 & 0.31 \\
reaction time & 67.18 & 81.40 & 66.39 & 139.78 & 76.55 & 68.17 & 71.37 & 76.71 & 55.58 & 67.74 \\
\hline
\end{tabular}
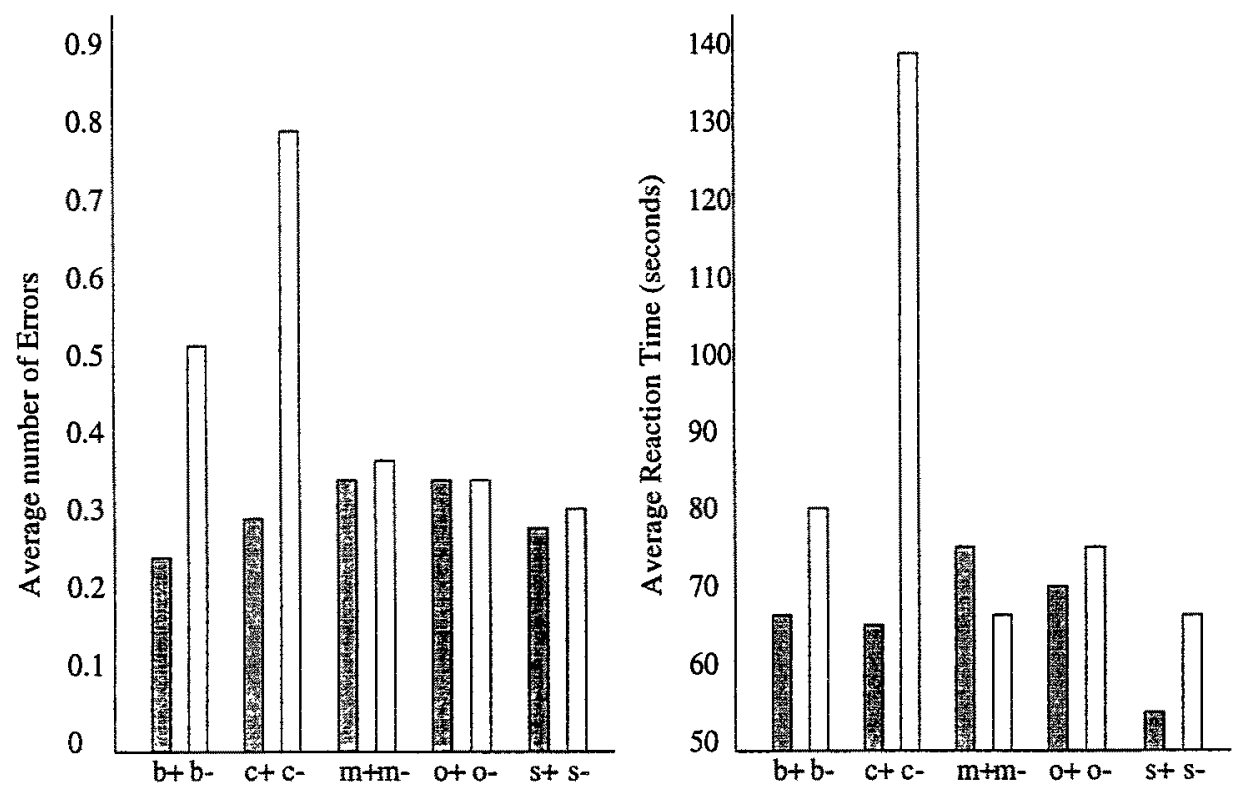

Fig. 3. The average reaction time and average number of errors for each graph drawing.

\subsection{Prioritising the Aesthetics}

To determine the relative effect of the aesthetics, and attempt to place a priority ordering on their importance, both the set of + drawings and the set of drawings needed to be tested for the overall effect of the aesthetics. Those sets of drawings for which the effect of the aesthetics were significant were then subject to a Tukey's pairwise comparison [9] to determine which aesthetics differed significantly from one another. 
Results. The $2 \times 5$ within-subject analysis of variance showed that:

- The main effect of the aesthetics dimension was significant for both errors $\left(F_{4,216}=4.16, \alpha=.05\right)$ and reaction time $\left(F_{4,216}=28.49, \alpha=.05\right)$.

\section{The - drawings:}

- The simple effects of the five different aesthetics were significant for the error data $\left(F_{4,216}=9.60, \alpha=025\right)$.

The Tukey's WSD pairwise comparisons procedure showed that, for the error data, crosses were significantly different from all other aesthetics: for bends $\left(F_{5,216}=9.11, \alpha=.05\right)$, minimum angle $\left(F_{5,216}=22.05, \alpha=.05\right)$, orthogonality $\left(F_{5,216}=24.20, \alpha=.05\right)$, symmetry $\left(F_{5,216}=30.01, \alpha=.05\right)$. There were no other significant pairwise differences.

- The simple effects of the five different aesthetics were significant for reaction time $\left(F_{4,216}=50.89, \alpha=.025\right)$.

The Tukey's WSD pairwise comparisons procedure showed that, for the reaction time data, crosses were significantly different from all other aesthetics: for bends $\left(F_{5,216}=95.09, \alpha=.05\right)$, minimum angle $\left(F_{5,216}=143.07, \alpha=.05\right)$, orthogonality $\left(F_{5,216}=110.98, \alpha=.05\right)$, symmetry $\left(F_{5,216}=144.79, \alpha=.05\right)$. There were no other significant pairwise differences.

\section{The + drawings:}

- The simple effects of the five different aesthetics were not significant for the error data $\left(F_{4,216}=1.02, \mathrm{NS}\right)$.

- The simple effects of the five different aesthetics were significant for the reaction time data $\left(F_{4,216}=4.68, \alpha=.025\right)$.

The Tukey's WSD pairwise comparisons procedure showed that, for the reaction time data, symmetry was significantly different from the minimum angle $\left(F_{5,216}=17.14, \alpha=.05\right)$, and orthogonality $\left(F_{5,216}=9.72, \alpha=.05\right)$.

\subsection{Analysis}

The error chart in Fig. 3 shows that the average number of errors for the versions of the drawings (i.e., the "difficult" drawings) was greater than the average number of errors for the + versions, in all cases except orthogonality when the averages were the same. The statistical analysis shows that the level of difficulty of the drawings was only significant for both bends and crosses.

The Tukey's pairwise comparison for the error data showed that the average number of errors for the c- drawing was significantly greater than the errors in the other - versions of the aesthetics, and that there were no significant pairwise orderings for the + drawings. 
The reaction time chart in Fig. 3 shows that - versions of the bends, crosses, orthogonality and symmetry drawings all took longer than the + versions. The statistical analysis shows that the level of difficulty of the drawings was only significant for both crosses and symmetry. The unexpected reversal of average reaction time for the two minimum angle drawings is not significant, and can therefore be attributed to chance.

The Tukey's pairwise comparison for the reaction time data showed that the c-drawing took significantly more time than all the other - versions of the aesthetics. In addition, the $\mathbf{s}+$ drawing took significantly less time than the minimum angle $\mathbf{m}+$ and orthogonality o+ drawings.

\subsection{Discussion}

There is no doubt that the evidence is overwhelmingly in favour of crosses as being the aesthetic that affects human relational graph reading the most, as suggested by the results of the two Tukey pairwise comparison tests performed on the - drawings. The effect of crosses was not noticeable, however, in the + drawings, implying that crosses are only more problematic than the other aesthetics when there are a large number of them.

The results of the other aesthetics are more ambivalent: the bends and symmetry hypotheses were supported either for reaction time or errors, but not both. Orthogonality and minimum angle had no effect on the subjects' relational graph reading at all. The Tukey test for the reaction time data for the + drawings showed that symmetry took significantly less time than the minimum angle and orthogonality, suggesting that symmetry only has a more positive effect than the other aesthetics when it is at a maximum value.

An unusual result was that for the easy drawings, the different aesthetics had no significant effect on the number of errors (even though there was an effect on reaction time). This suggests that the subjects tended to give correct answers on all aesthetics if the drawings were easy, but they used all the time necessary, requiring different amounts of time for the different aesthetics. On the other hand, for the difficult drawings, subjects took the amount of time necessary (which differed for the different aesthetics), but the difficulty of the drawings meant that the number of errors was also differentially affected for different aesthetics.

In interpreting the above result, errors can be interpreted as a measure of the amount of processing required to get the question right, while reaction time can be interpreted as a measure the perceptual processing and comprehension of the drawing. 
Limitations. It is common knowledge that all experiments are limited by their parameters, and that the results of any experiment should always be interpreted with respect to the experimental limitations [7]: this is an inevitable consequence of the controlled experimental method. These results can therefore only be interpreted within the context of the graph and tasks specified. There may also be a generalisability restriction on the nature of the subjects, who were all computer scientists, although as a within-subject analysis was performed, any variations in expertise were controlled.

\section{Conclusions}

These aim of these empirical tests was to indicate to the designers of graph drawing algorithms the most effective aesthetics to use from the point of view of human reading of relational information. The results show that there is strong evidence to support minimising crosses, and weaker evidence for minimising the number of bends and maximising perceptual symmetry. Maximising the orthogonal structure of the drawing; and maximising the minimum angles between edges leaving a node, appear to have little effect.

There is still much work to do in this area: this experiment has only considered the relational reading of graph drawings; and different results may be forthcoming from experiments that require an interpretive reading of graph drawings in the context of application domains. For example, testing the effect of the different aesthetics when the graph drawings represent object-oriented design diagrams or data-flow diagrams may produce different results.

In addition, another possible study could consider the relational understandability of graph drawings generated by different layout algorithms which aim to maximise the effect of particular aesthetics: it would be interesting to see whether the results obtained from that experiment are compatible with the results of the study reported here.

\section{Acknowledgements}

I am grateful to Robert Cohen (who assisted with the initial experimental definition), to Murray James (who designed and developed SAGE), to David Leonard (who helped define and implement the aesthetic metrics), to Julie McCreddon (who assisted extensively with the statistical analysis), and the Australian Research Council, which funded this work. 


\section{References}

1. C. Batini, M. Talamo; and R. Tamassia. Computer aided layout of entityrelationship diagrams. Journal of Systems and Software, 4:163-173, 1984.

2. S. Bhanji, H.C. Purchase, R.F. Cohen, and M.I. James. Validating graph drawing aesthetics: A pilot study. Technical Report 336, University of Queensland Department of Computer Science, 1995.

3. J. Blyth, C. McGrath, and D. Krackhardt. The effect of graph layout on inference from social network data. In F. Brandenburg, editor, Proceedings of Graph Drawing Symposium 1995. Springer-Verlag; Passau, Germany, 1995. Lecture Notes in Computer Science, 1027.

4. G. Booch. Object-Oriented Design. Benjamin-Cummings, 1990.

5. P. Eades. A heuristic for graph drawing. Congressus Numerantium, 42:149-160, 1984.

6. D. Ferrari and L. Mezzalira. On drawing a graph with the minimum number of crossings. Technical Report 69-11, Istituto di Elettrotecnica ed Elettronica, Politecnico di Milano, 1969.

7. R. Gottsdanker. Experimenting in Psychology. Prentice-Hall, 1978.

8. M. Himsolt. GraphEd user manual. Universität Passau, 1990.

9. P.R. Hinton. Statistics Explained. Routledge, 1995.

10. R. Lipton, S. North, and J. Sandberg. A method for drawing graphs. In Proc. ACM Symp. on Computational Geometry, pages 153-160, 1985.

11. H.C. Purchase, R.F. Cohen, and M. James. An experimental study of the basis for graph drawing algorithms. ACM Journal of Experimental Algorithmics, 2(4), 1997.

12. H.C. Purchase and D. Leonard. Graph drawing aesthetic metrics. Technical Report 361, University of Queensland Department of Computer Science, 1996.

13. R. Tamassia. On embedding a graph in the grid with the minimum number of bends. SIAM J. Computing, 16(3):421-444, 1987.

14. R. Tamassia, G. Di Battista, and C. Batini. Automatic graph drawing and readability of diagrams. IEEE Transactions on Systems, Man and Cybernetics, SMC18(1):61-79, 1988.

15. H. Trickey. Drag: A graph drawing system. In Proc. Int. Conf. on Electronic Publishing, pages 171-182. Cambridge University Press, 1988. 